\title{
Dynamic Scaling of Magnetic Flux Noise Near the KTB Transition in Overdamped Josephson Junction Arrays
}

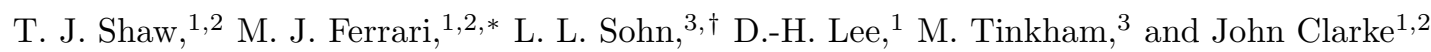 \\ ${ }^{1}$ Department of Physics, University of California, Berkeley, California 94720 \\ ${ }^{2}$ Materials Sciences Division, Lawrence Berkeley Laboratory, University of California, Berkeley, California 94720 \\ ${ }^{3}$ Department of Physics and Division of Applied Sciences, Harvard University, Cambridge, Massachussetts 02138 \\ (October 17, 2018) \\ We have used a dc Superconducting QUantum Interference Device to measure the magnetic \\ flux noise generated by the equilibrium vortex density fluctuations associated with the Kosterlitz- \\ Thouless-Berezinskii (KTB) transition in an overdamped Josephson junction array. At temperatures \\ slightly above the KTB transition temperature, the noise is white for $f<f_{\xi}$ and scales as $1 / f$ for \\ $f>f_{\xi}$. Here $f_{\xi} \propto \xi^{-z}$, where $\xi$ is the correlation length and $z$ is the dynamic exponent. Moreover, \\ when all frequencies are scaled by $f_{\xi}$, data for different temperatures and frequencies collapse on to \\ a single curve.
}

PACS numbers: 74.50.+r, 74.40.+k 
Arrays of Josephson junctions have been used extensively as a model system to study the effects of order parameter phase fluctuations on the superconducting transition in two dimensions. Such arrays can be fabricated with a high degree of uniformity and their relevant parameters can be accurately determined. It is widely accepted that the zero field transition is described by the Kosterlitz-Thouless- Berezinskii (KTB) theory [1 1 - and its extension to non-zero frequency [5 7]. According to this theory, phase coherence is established throughout the sample below a temperature $T_{\mathrm{KTB}}$, and the system is superconducting. For temperatures above $T_{\mathrm{KTB}}$ but below the bulk transition temperature, even though individual islands are superconducting the array is not. The thermal excitations, vortices and antivortices, that trigger this phase transition are topological defects of the order parameter. Below $T_{\mathrm{KTB}}$ vortices and antivortices bind in pairs to produce a vortex dielectric, while above $T_{\mathrm{KTB}}$ the pairs dissociate to form a vortex plasma. In the vortex plasma phase, one can identify a characteristic length, $\xi$, as the average separation between free vortices; as $T \rightarrow T_{\mathrm{KTB}}^{+}, \xi$ diverges. Thermal fluctuations that perturb the vortex density away from its equilibrium value relax through some local dynamic process. Thus, associated with the characteristic length $\xi$ there is a characteristic time $\tau$ (or an inverse characteristic frequency $\left.f_{\xi}^{-1} \propto \tau\right)$ corresponding to the time required for the disturbance to propagate across the distance $\xi$. As $\xi$ diverges, so does $\tau\left(f_{\xi}^{-1}\right)$, signifying critical slowing down. In general $\tau=\tau_{0}\left(\xi / \xi_{0}\right)^{z}$, where the exponent $z$ depends on the dynamics of the relaxation and $\tau_{0}$ and $\xi_{0}$ are non-universal time and length scales characteristic to the specific sample. For simple diffusion, $z=2$.

Previous experimental studies have involved both electrical resistance [8 16] and two-coil mutual inductance [17] techniques, both of which apply an external force to the system and are generally confined to a specific frequency. Because the transition to the resistive state is determined by the dissociation of vortex pairs by thermal fluctuations, these external forces, which also dissociate pairs, affect one's ability to study the intrinsic critical phnomena near the true thermodynamic transition temperature. In this paper we employ a non-invasive probe to study the transition in equilibrium. Specifically, we measure the spectral density of magnetic flux noise [18], $S_{\Phi}(f)$, over a frequency range of more than five decades. We find that $S_{\Phi}(f)$ is white for $f<f_{\xi}$ and scales as $1 / f$ for $f>f_{\xi}$. In addition, by plotting $f S_{\Phi}(f)$ versus $f / f_{\xi}$ we show that the data collapse in a manner consistent with dynamic scaling.

The $1 \mathrm{~mm} \times 1 \mathrm{~mm}$ array 19 consists of $0.2 \mu \mathrm{m}$-thick cross-shaped niobium islands patterned on top of a 0.3 $\mu$ m-thick copper film [Fig. 1(a)]. The islands form a square array with a lattice constant of $10 \mu \mathrm{m}$, and the junctions are $4 \mu \mathrm{m}$ wide and $2 \mu \mathrm{m}$ long. The measurement apparatus, originally used to study vortex mo- tion in high-temperature superconductors [20], involves a Nb-based Superconducting QUantum Interference Device (SQUID) attached to a cold stage inside a vacuum can surrounded by liquid ${ }^{4} \mathrm{He}$. A mu-metal shield reduces the static magnetic field to less than $1 \mu \mathrm{T}$. The array, equipped with current and voltage leads, is mounted a distance $d(<100 \mu \mathrm{m})$ away on a variable temperature stage. The SQUID is a square washer with inner and outer dimensions $\ell_{i}=180 \mu \mathrm{m}$ and $\ell_{o}=900 \mu \mathrm{m}$ [Fig. 1(b)], and is operated in a flux-locked loop. The output signal is proportional to the change in magnetic flux through the SQUID induced by vortex motion in the array.

The inset to Fig. 2 shows the differential resistance, $d V / d I$, of the array versus temperature, $T$, measured at zero bias current with an rms current of $10 \mu \mathrm{A}$ at a frequency of $47 \mathrm{~Hz}$. The initial drop in the resistance at approximately $T=8 \mathrm{~K}$ marks the bulk transition temperature of the niobium islands. As the temperature is lowered, a resistive plateau develops and is followed by a second precipitous drop, which is the KTB transition. These data are similar to those obtained in previous experiments 8 16. To obtain the average critical current per junction, $i_{c}(T)$, we measure $d V / d I$ as a function of the static bias current. We take the current at which $d V / d I$ is a maximum as $N i_{c}(T)$, where $N$ is the number of junctions across the width of the array [21,22].

Figure 2 shows the spectral density of flux noise, $S_{\Phi}(f)$, for 15 temperatures above $T_{\mathrm{KTB}}$. At each temperature $S_{\Phi}(f)$ is white for $f<f_{\xi}(T)$ and $\propto 1 / f$ for $f>f_{\xi}(T)$. We define $f_{\xi}(T)$ as the intersection of lines through the white and $1 / f$ noise regions as shown in Fig. 2 for $T=1.978 \mathrm{~K}$. Qualitatively, we understand the difference between the two frequency regimes as follows. For $f>f_{\xi}$ we probe the system at a time scale shorter than that required for vortex density disturbances to travel over the correlation length. Thus, the system appears to be critical. On the other hand, for $f<f_{\xi}$ the time scale
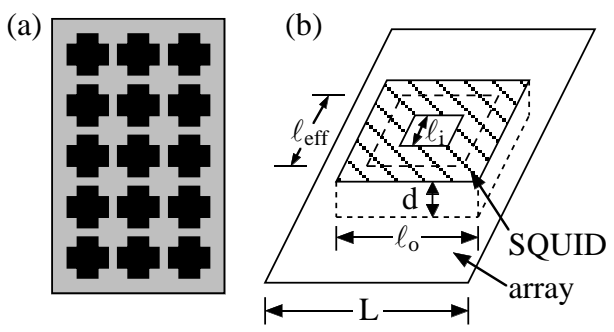

FIG. 1. (a) Schematic of array. Crosses are niobium islands; area between crosses is copper film. (b) Schematic representation of SQUID, with inner and outer dimensions $\ell_{i}$ and $\ell_{o}$, a distance $d$ from array; dashed square has area $\ell_{\text {eff }}^{2}=\ell_{i} \ell_{o}$. 


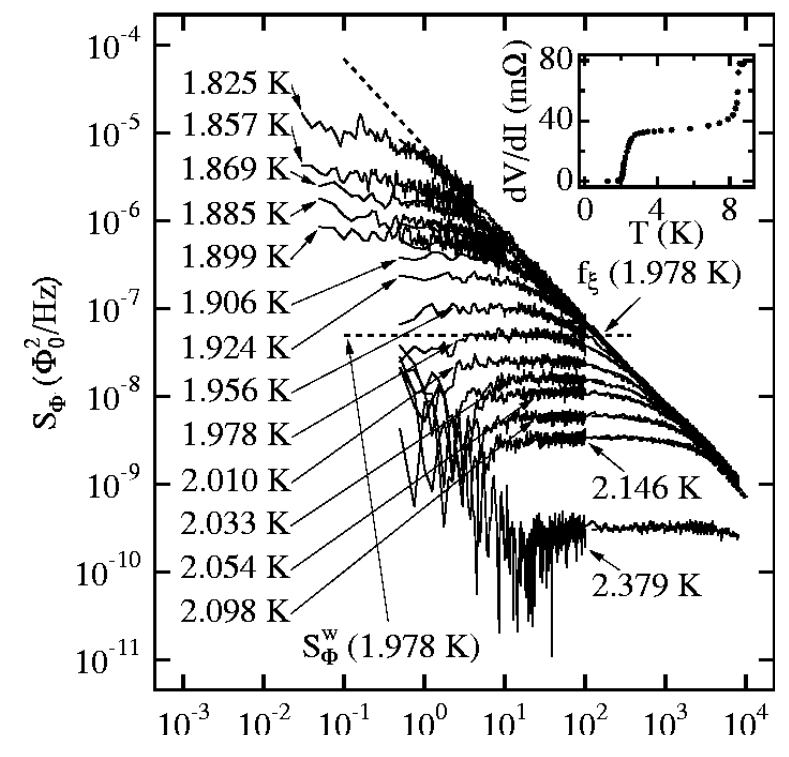

FIG. 2. Spectral density of magnetic flux noise, $S_{\Phi}(f)$, versus frequency for 15 temperatures above $T_{\mathrm{KTB}}$; scatter at higher temperatures is due to subtraction of SQUID noise. Dashed lines have slope -1 and 0 . Inset shows $d V / d I$ versus $T$.

is longer than the characteristic relaxation time, so that the system appears disordered. In this picture, the distinctions between the $1 / f$ (critical) and white (disordered) power spectra reflect the fundamental differences in the relaxation dynamics of these two very different thermodynamic states. It is worthwhile to point out that theories based on the two-dimensional XY model and time-dependent-Ginzburg-Landau dynamics predict a $1 / f^{2}$ behavior for $f>f_{\xi}$ [23,24]. This is in marked contrast to the $1 / f$ behavior observed in our experiment.

To interpret our data, we now present a brief discussion of a scaling theory for the flux noise measurements. In our geometry [Fig. 1(b)], $\hat{h}$ is the direction perpendicular to the plane $(h=0)$ defined by the array. We denote a three-dimensional vector by $(\vec{x}, h)$, where $\vec{x}$ is the component of the vector in the plane of the array. In the absence of the SQUID, the perpendicular component of the magnetic field, $B_{\perp}$, induced at point $(\vec{x}, h)$ at time $t$ by a vortex density distribution $\rho_{v}\left(\vec{x}^{\prime}, t\right)$ at $h=0$ is

$$
B_{\perp}(\vec{x}, h ; t)=\Phi_{0} \int d^{2} x^{\prime} K\left(\vec{x}-\vec{x}^{\prime}, h\right) \rho_{v}\left(\vec{x}^{\prime}, t\right) .
$$

Here $\Phi_{0}=h c / 2 e$ and $K\left(\vec{x}-\vec{x}^{\prime}, h\right) \propto h /\left(\left|\vec{x}-\vec{x}^{\prime}\right|^{2}+h^{2}\right)^{3 / 2}$ for $\left|\vec{x}-\vec{x}^{\prime}\right|^{2}+h^{2} \gg a^{2}$ [23], where $a$ is the lattice constant of the array. The total flux, $\Phi(t)$, detected by the SQUID is $\Phi(t)=\int d^{2} x B_{\perp}(\vec{x}, h=d ; t)$, where the integral is performed over the effective area of the SQUID, $\ell_{\mathrm{eff}}^{2}=\ell_{i} \ell_{o}$. In fact, magnetic field lines produced by vortices near the SQUID are distorted by the presence of the superconducting washer, modifying the form of $K$. However, we do not expect this modification to change the scaling dimension of $K$ from two (that is, if we replace $\vec{x}, \vec{x}^{\prime}, d$ by $\vec{x} / \xi, \vec{x}^{\prime} / \xi, d / \xi$, we still expect $K\left(\left|\vec{x}-\vec{x}^{\prime}\right|, d\right)$ to become $\xi^{-2} K\left(\left(\vec{x}-\vec{x}^{\prime}\right) / \xi, d / \xi\right)$ so that our scaling arguments remain valid. Thus the flux-flux correlation function, $C_{\Phi}(t) \equiv\langle\Phi(t) \Phi(0)\rangle$, is

$$
\begin{aligned}
C_{\Phi}(t)= & \Phi_{0} \int d^{2} x d^{2} y d^{2} x^{\prime} d^{2} y^{\prime} K\left(\vec{x}-\vec{x}^{\prime}, d\right) \\
& \times K\left(\vec{y}-\vec{y}^{\prime}, d\right)\left\langle\rho_{v}\left(\vec{x}^{\prime}, t\right) \rho_{v}\left(\vec{y}^{\prime}, 0\right)\right\rangle .
\end{aligned}
$$

Here, \langle\rangle denotes the thermodynamic average, and the unprimed integrals are taken over the effective area of the SQUID. Next, we assume a scaling ansatz for the vortex density-density correlation function:

$$
\left\langle\rho_{v}\left(\vec{x}^{\prime}, t\right) \rho_{v}\left(\vec{y}^{\prime}, 0\right)\right\rangle=\xi^{-4} F_{1}\left(t / \tau,\left|\vec{x}^{\prime}-\vec{y}^{\prime}\right| / \xi, L / \xi\right) .
$$

Here we have used the fact that the scaling dimension of the vortex density is two at the KTB transition (that is, $\rho_{v} \propto \xi^{-2}$ ). In Eq. (3), $F_{1}$ is a scaling function and $L$ is the smaller dimension of the array. For Josephson junction arrays,

$$
\begin{array}{r}
\xi=\xi_{0} \exp \left(b / \sqrt{T^{\prime}-T_{\mathrm{KTB}}^{\prime}}\right), \\
T^{\prime} \equiv k_{B} T / E_{J}(T)=2 e k_{B} T / \hbar i_{c}(T),
\end{array}
$$

and $E_{J}(T)$ is the Josephson coupling energy per junction [17. If we substitute the scaling ansatz, Eq. (3), into Eq. (2) and perform proper rescaling of the integration variables, one can show that

$$
C_{\Phi}(t)=\Phi_{0}^{2} F_{2}\left(t / \tau, d / \xi, L / \xi, \ell_{\mathrm{eff}} / \xi\right),
$$

where $F_{2}$ is a new scaling function. The noise spectrum, $S_{\Phi}(f)$, is defined as $S_{\Phi}(f)=\int d t \exp (i 2 \pi f t) C_{\Phi}(t)$. Substituting Eq. (5) in this expression, we find

$$
f S_{\Phi}(T)=\Phi_{0}^{2} F\left(f / f_{\xi}, d / \xi, L / \xi, \ell_{\mathrm{eff}} / \xi\right) .
$$

Here $F$ is another scaling function, the form of which we determine from a subsequent data collapse.

If we ignore the dependence on $d / \xi, L / \xi$, and $\ell_{\mathrm{eff}} / \xi$, Eq. (6) predicts

$$
f S_{\Phi}(T)=\Phi_{0}^{2} F\left(\left(f / f_{0}\right) \exp \left(b z / \sqrt{T^{\prime}-T_{\mathrm{KTB}}^{\prime}}\right)\right),
$$

where we have explicitly put in the temperature dependence of $f_{\xi}$. In Eq. (7), $T_{\mathrm{KTB}}$ and $b z$ are unknown. To determine $T^{\prime}$, we fit $i_{c}(T)$ between $T=$ $1.52 \mathrm{~K}$ and $T=1.93 \mathrm{~K}$ using the expected temperature dependence $i_{c}(T)=i_{c}(0) \exp (-\alpha \sqrt{T})$ [25], finding $i_{c}(0)=0.133 \mathrm{~A}$ and $\alpha=9.14 \mathrm{~K}^{-1 / 2}$, and extrapolate to higher temperatures. Equation (7) predicts that by choosing the correct values for $T_{\mathrm{KTB}}$ and $b z$ and plotting $f S_{\Phi}(f)$ versus the scaled frequency $f / \tau_{0} f_{\xi}=$ $f \exp \left(b z / \sqrt{T^{\prime}-T_{\mathrm{KTB}}^{\prime}}\right)$, we should obtain a collapse of 
the raw data onto a single curve. Our procedure for data collapsing is to make iterative changes in $b z$ and $T_{\mathrm{KTB}}$ until the best data collapse is obtained. The final result is shown in Fig. 3, where the fitting parameters were $b z=4$ and $T_{\mathrm{KTB}}=1.63 \mathrm{~K}$. The quality of the data collapse is strongly affected by the choice of $T_{\mathrm{KTB}}$ but relatively weakly by the choice of $b z$. One can compensate an increase (decrease) of $T_{\mathrm{KTB}}$ of a few $\mathrm{mK}$ by a decrease (increase) in $b z$ of about $10 \%$. However, changes in $T_{\mathrm{KTB}}$ beyond a few $\mathrm{mK}$ or changes in $b z$ of more than about $10 \%$ always result in a lower quality data collapse. We can obtain a more accurate estimate of $b z$ through the temperature dependence of $f_{\xi}(T)$. Using Eqs. (4) and $f_{\xi}(T)=f_{0}\left[\xi(T) / \xi_{0}\right]^{-z}$, we expect a straight line of slope $-b z$ on a plot of $\ln \left(f_{\xi}\right)$ versus $1 / \sqrt{T^{\prime}-T_{\mathrm{KTB}}^{\prime}}$. Inset (a) to Fig. 3, using $T_{\mathrm{KTB}}=1.63 \mathrm{~K}$, shows the resultant straight line which yields $b z=4.10 \pm 0.04$ and $f_{0}=(2.1 \pm 0.3) \times 10^{6} \mathrm{~Hz}$.

We note that although $f_{0}$ is in the megahertz frequency range, the exponential dependence of $f_{\xi}$ on $T^{\prime}-T_{\mathrm{KTB}}^{\prime}$ implies that $f_{\xi}$ is reduced to the order of $1 \mathrm{~Hz}$ even when $T$ is about $0.2 \mathrm{~K}$ above $T_{\mathrm{KTB}}$. This sensitivity is illustrated by the fact that the apparent transition temperature, $T_{\mathrm{KTB}}(47 \mathrm{~Hz})$, associated with the sharp rise in $d V / d I$ measured at $47 \mathrm{~Hz}$ (inset, Fig. 2) is substantially higher than the static value $T_{\mathrm{KTB}}=1.63 \mathrm{~K}$ inferred from our noise data. However, these values are quite consistent since $T_{\mathrm{KTB}}(47 \mathrm{~Hz})$ is the temperature at which $f_{\xi}=47 \mathrm{~Hz}$. Using the above values of $T_{\mathrm{KTB}}, f_{0}$, and $b z$, we predict $T_{\mathrm{KTB}}(47 \mathrm{~Hz})=1.94 \mathrm{~K}$, in good agreement with the $d V / d I$ data (inset, Fig. 2).

In addition to finding $b z$, we can extract the critical exponent $z$ in the following manner. At low frequencies, a two-dimensional sample with a linear conductivity $\sigma$ produces white noise with spectral density $S_{\Phi}^{w} \propto k_{B} T \sigma$. In the KTB regime for $f \ll f_{\xi}, \sigma$ is proportional to $\left\langle\rho_{v}\right\rangle^{-1}=\xi^{2}$, reflecting the intuitive idea that the low frequency conductivity is inversely proportional to the

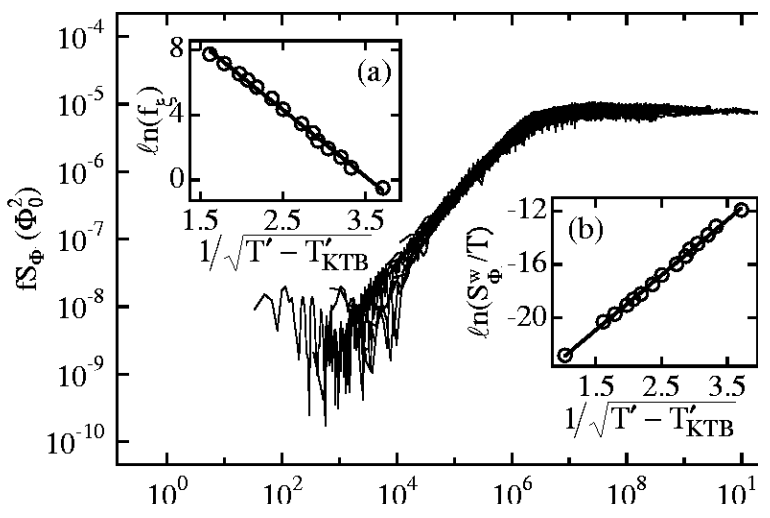

FIG. 3. $f S_{\Phi}(f)$ versus $f / \tau_{0} f_{\xi}$ for flux noise shown in Fig. 2. $T_{\mathrm{KTB}}=1.63 \mathrm{~K}, b z=4$. Insets show $\ln \left(f_{\xi}\right)$ versus $1 / \sqrt{T^{\prime}-T_{\mathrm{KTB}}^{\prime}}$ with line of slope -4.10 and $\ln \left(S_{\Phi}^{w} / T\right)$ versus $1 / \sqrt{T^{\prime}-T_{\mathrm{KTB}}^{\prime}}$ with line of slope 4.13 . density of free vortices. Therefore, for $f \ll f_{\xi}$,

$$
S_{\Phi}^{w} \propto k_{B} T \xi_{0}^{2} \exp \left(2 b / \sqrt{T^{\prime}-T_{\mathrm{KTB}}^{\prime}}\right) .
$$

To test Eq. (8), we define $S_{\Phi}^{w}$ as the horizontal line drawn through the low frequency data at each temperature, as shown for $T=1.978 \mathrm{~K}$ in Fig. 2, and plot $\ln \left(S_{\Phi}^{w} / T\right)$ versus $1 / \sqrt{T^{\prime}-T_{\mathrm{KTB}}^{\prime}}$. Inset (b) to Fig. 3, using $T_{\mathrm{KTB}}=1.63 \mathrm{~K}$, shows the resultant straight line which yields $2 b=4.13 \pm 0.04$. Combining this result with the value $b z=4.10 \pm 0.04$ from the temperature dependence of $f_{\xi}$ yields the dynamic exponent $z=1.98 \pm 0.03$.

In summary, we have used measurements of magnetic flux noise to study the equilibrium KTB transition in an array of overdamped Josephson junctions. We emphasize that confirmation of the critical dynamics of the KTB transition requires measurement of frequency and temperature dependent properties such as those presented above. Using $b z=4$ and $T_{\mathrm{KTB}}=1.63 \mathrm{~K}$, we have shown that the data collapse over more than five decades in frequency, confirming the predictions of dynamic scaling. In addition, from the temperature dependences of $f_{\xi}$ and $S_{\Phi}^{w}$, we have extracted the dynamic exponent $z=$ $1.98 \pm 0.03$. Our experimental finding $S_{\Phi}(f) \propto 1 / f$ in the critical frequency regime is inconsistent with theoretical predictions based on time-dependent Ginzburg-Landau (TDGL) dynamics with the classical two-dimensional XY model [23,24]. The failure of this model is surprising since TDGL dynamics does predict $z=2$, in agreement with our extracted value. It is unclear to us why $S_{\Phi}(f, T)$ is independent of $d / \xi, L / \xi$, and $\ell_{\text {eff }} / \xi$. A possible cause may be that the data in Fig. 2 are in the regime $\xi(T)<\ell_{\text {eff }}<L$. Our evidence for this restriction is that at temperatures between $T_{\mathrm{KTB}}$ and $1.825 \mathrm{~K}$ (the lowest temperature for which data are shown in Fig. 2), we observe discrete jumps in the flux threading the SQUID. We interpret this behavior as the motion of a single vortex under the SQUID, implying that $\xi(T)>\ell_{\text {eff }}$, and conclude that $\xi(T)<\ell_{\text {eff }}$ for the temperatures referred to in Fig. 22. If we assume $\xi(T=1.825 \mathrm{~K})<\ell_{\mathrm{eff}}$, we deduce $\xi_{0}<0.2 \mu \mathrm{m}$ [Eqs. (田) , a value that is considerably smaller than the commonly accepted lore $\xi_{0}=a$. Setting $\xi_{0}<0.2 \mu \mathrm{m}$, we deduce $\xi<2 \mu \mathrm{m}$ for the highest temperature $(2.379 \mathrm{~K})$ data shown in Fig. 2. The fact that scaling persists to this temperaure where the correlation length is smaller than the lattice constant $a$ is somewhat disturbing. Further work is needed to resolve this issue, as well as the lack of dependence of $F$ on $d / \xi, L / \xi$, and $\ell_{\mathrm{eff}} / \xi$, and the $1 / f$ behavior of the flux noise in the critical regime.

In closing, we note that we have carried out similar measurements on two other arrays, one of square geometry and the other of triangular geometry, using the SQUID described above as well as two other SQUIDS of different geometry. When the apparatus was cooled in a magnetic field $(<1 \mu \mathrm{T})$, the data were similar to those 
described above with minor differences in the high frequency behavior. When a small field was applied, the noise data exhibited very different behavior. These additional measurements will be described elsewhere.

We thank Roger Koch for helpful discussions and Chris Lobb for sharing his constructive criticisms and knowledge of Josephson junction arrays with us. This work was supported by the Director, Office of Energy Research, Office of Basic Energy Sciences, Materials Science Division of the U. S. Department of Energy under contract number DE-AC03-76SF00098.

* Current address: BARRA, Berkeley, California, 94704.

$\dagger$ Current address: Department of Physics, Princeton University, Princeton, New Jersey, 08544.

[1] J. M. Kosterlitz and D. J. Thouless, J. Phys. C, 6, 1181 (1973).

[2] J. M. Kosterlitz, J. Phys. C, 7, 1046 (1974).

[3] V. L. Berezinskii, Zh. Eksp. Teor. Fiz. 59, 207 (1970) [Sov. Phys. JETP 32, 493 (1971)].

[4] C. J. Lobb, D. W. Abraham, and M. Tinkham, Phys. Rev. B 27, 150 (1983).

[5] B. I. Halperin and D. R. Nelson, J. Low Temp. Phys. 36, 599, (1979).

[6] V. Ambegaokar, B. I. Halperin, D. R. Nelson, and E. D. Siggia, Phys. Rev. B 21, 1806 (1980).

[7] S. R. Shenoy, J. Phys. C, 18, 5163 (1985).

[8] D. J. Resnick, J. C. Garland, J. T. Boyd, S. Shoemaker, and R. S. Newrock, Phys. Rev. Lett. 47, 1542 (1981).

[9] R. F. Voss and R. A. Webb, Phys. Rev. B 25, 3446 (1982).

[10] D. W. Abraham, C. J. Lobb, M. Tinkham, and T. M. Klapwijk, Phys. Rev. B 26, 5268 (1982).

[11] R. A. Webb, R. F. Voss, G. Grinstein, and P. M. Horn, Phys. Rev. Lett. 51, 690 (1983).

[12] D. Kimhi, F. Leyvraz, and D. Ariosa, Phys. Rev. B 29, 1487 (1984).

[13] R. K. Brown and J. C. Garland, Phys. Rev. B 33, 7827 (1986).

[14] B. J. van Wees, H. S. J. van der Zant, and J. E. Mooij, Phys. Rev. B 35, 7291 (1987).

[15] J. P. Carini, Phys. Rev. B 38, 63 (1988).

[16] H. S. J. van der Zant, H. A. Rijken, and J. E. Mooij, Phys. Rev. B 82, 67 (1991).

[17] Ch. Leemann, Ph. Lerch, G.-A. Racine, and P. Martinoli, Phys. Rev. Lett. 56, 1291 (1986).

[18] Previous SQUID-based measurements found $S_{\Phi}(f) \propto$ $1 / f$ for $T \leq T_{\mathrm{KTB}}$, but were not able to resolve any crossover to white noise for $T>T_{\mathrm{KTB}}$ [Ph. Lerch, Ch. Leemann, R. Théron, and P. Martinoli, Helv. Phys. Acta 65, 389 (1992)].

[19] L. L. Sohn, Ph.D. Thesis, Harvard University, 1992.

[20] M. J. Ferrari, Mark Johnson, F. C. Wellstood, J. J. Kingston, T. J. Shaw, and John Clarke, J. Low Temp. Phys. 94, 15 (1994).
[21] V. Ambegaokar and B. I. Halperin, Phys. Rev. Lett. 22, 1364 (1969).

[22] C. M. Falco, W. H. Parker, S. E. Trulliger, and P. K. Hansma, Phys. Rev. B 10, 1867 (1974).

[23] D.-H. Lee, unpublished.

[24] J. Houlrik, A. Jonsson, and P. Minnhagen, Phys. Rev. B 50, 3953 (1994); A. Jonsson and P. Minnhagen, Phys. Rev. Lett. 73, 3576 (1994).

[25] P. G. de Gennes, Rev. Mod. Phys. 36, 225 (1964). 